\title{
Efficacy of Frovatriptan vs. Other Triptans in Weekend Migraine: Pooled Analysis of Three Double-Blind, Randomized, Crossover, Multicenter Studies
}

\author{
Carlo Lisotto $^{1^{*}}$, Lidia Savi ${ }^{2}$, Lorenzo Pinessi ${ }^{2}$, Mario Guidotti $^{3}$, Stefano Omboni ${ }^{4}$ and Giorgio Zanchin ${ }^{5}$ \\ ${ }^{1}$ Department of Neurology, Azienda Ospedaliera Santa Maria degli Angeli, Pordenone, Italy \\ ${ }^{2}$ Neurology II, Headache Center, Department of Neurosciences, University of Turin, Italy \\ ${ }^{3}$ Department of Neurology, Valduce Hospital, Como, Italy \\ ${ }^{4}$ Italian Institute of Telemedicine, Varese, Italy \\ ${ }^{5}$ Headache Center, Department of Neurosciences, University of Padua, Italy
}

*Corresponding author: Dr. Carlo Lisotto, Headache Center, Department of Neurology, Azienda Ospedaliera Santa Maria degli Angeli, Via Montereale 24, 33170 Pordenone, Italy, Tel: +390434841420; Fax: +390434841611; E-mail: carlo.lisotto@aopn.sanita.fvg.it

Received date: Apr 15, 2014, Accepted date: May 22, 2014, Published date: May 29, 2014

Copyright: (c) 2014 Lisotto C, et al. This is an open-access article distributed under the terms of the Creative Commons Attribution License, which permits unrestricted use, distribution, and reproduction in any medium, provided the original author and source are credited.

\begin{abstract}
Background: Migraine attacks usually occur at any time, in particular either during days off or workdays. Limited evidence is available on the efficacy of antimigraine drugs in patients whose attacks occur on weekends.

Objective: To evaluate the efficacy of four different triptans in weekend vs. workday migraine attacks through a pooled analysis of individual data of three randomized, double-blind, crossover studies.

Methods: Subjects with a history of migraine with or without aura were randomized to frovatriptan $2.5 \mathrm{mg}$ or rizatriptan $10 \mathrm{mg}$ (study 1), frovatriptan $2.5 \mathrm{mg}$ or zolmitriptan $2.5 \mathrm{mg}$ (study 2), frovatriptan $2.5 \mathrm{mg}$ or almotriptan $12.5 \mathrm{mg}$ (study 3). Each patient was requested to treat up to 3 attacks with each drug during both 3 month periods, as established by the crossover trial protocol. In this retrospective analysis the migraine attacks occurring on workdays were differentiated from those occurring on weekends. The efficacy of the drugs during weekends and workdays was compared.
\end{abstract}

Results: Of the 346 intention-to-treat population patients, $188(54 \%)$ had migraine attacks also on weekends. Overall, a total of 569 attacks occurred during weekends and 1,281 during workdays. Proportion of pain-free at 2 hours did not significantly differ between weekend and workday attacks for frovatriptan ( $26 \%$ vs. $27 \%$ ) as well as for comparators $(34 \%$ vs. $32 \%)$. Headache relief episodes were also similarly represented between weekend and nonweekend attacks (frovatriptan: $40 \%$ vs. $42 \%$ and comparators: $49 \%$ vs. $43 \%$, p=NS). Conversely, the relapse rate within 48 hours for weekend attacks compared to workday attacks was significantly lower with frovatriptan $(17 \%$ vs. $30 \%, p<0.05$ ), while this finding was not observed for comparators (weekends $34 \%$ vs. workdays $40 \%, p=N S$ ).

Conclusions: Our study provides the first evidence that frovatriptan represents a particularly favorable option for treating weekend migraine attacks.

Keywords: Migraine Headache; Frovatriptan; Rizatriptan; Zolmitriptan; Almotriptan; Weekend Migraine; Relapse.

\section{Introduction}

Approximately $30 \%$ of patients with a diagnosis of migraine or, less frequently, tension-type headache, claim to have their attacks mainly on weekends, particularly in case of migraine without aura [1,2]. It has been a matter of debate in recent years whether days off represent a possible trigger for headache, with some studies showing a clear relationship $[3,4]$ and other surveys failing to confirm this association [5-7].

Psychological factors seem to play an important role in the pathophysiology and clinical development of weekend migraine and some authors in the past have postulated that a psychic tension component might be superimposed to the vascular component in this subtype of migraine [8]. Although weekend migraine has been a poorly investigated condition over the years and specific evidence of drug efficacy for treatment of this condition is lacking, there is no reason to doubt that current drug options for migraine treatment can also be effective for weekend headache.

Selective serotonin 5-HT1B/1D receptor agonists (the triptans) have been shown to be effective acute migraine drugs, and are currently recommended as first-line treatment for moderate-to-severe migraine, or for mild-to-moderate migraine attacks which have not responded to adequate doses of simple analgesics or non-steroidal antiinflammatory drugs [9-12]. In an observational prospective survey regarding 3415 patients it was found out that more than $5 \%$ of all migraineurs have their attacks mostly on weekends. The subjects with at least $75 \%$ of all their migraine attacks occurring on weekends, with a frequency ranging from 1 attack every 2 months to 4 attacks per month, were arbitrarily considered as patients suffering from weekend migraine. Up to now possible diagnostic criteria for weekend migraine are lacking and have never been proposed. Anyway, weekend migraine 
was unexpectedly found to be more common in males than in females (52.6\% vs. $47.4 \%)$; the attacks were reported to be severe in intensity and markedly disabling in most cases, responding only to triptans in over $50 \%$ of the subjects [13].

Frovatriptan is the newest addition to the triptan class, developed in order to provide a compound with a clinical potential for a long duration of action and a low likelihood of side effects and drug interactions $[14,15]$. Recently, three direct comparative, prospective, double-blind, randomized, crossover studies have compared the efficacy and safety of frovatriptan versus other second-generation triptans, such as rizatriptan [16], zolmitriptan [17] and almotriptan [18]. The study results showed a similar efficacy of the four triptans in the early treatment (within 2 hours) of migraine, but a lower rate of relapse, and thus a better sustained relief, with frovatriptan. Retrospective analyses of the same studies demonstrated the good efficacy profile of frovatriptan also in other subtypes of migraine, such as menstrually related migraine [19-22] and migraine with aura [23].

In this paper we report on the results of a retrospective analysis performed in patients having migraine attacks also on weekends, who were enrolled in the three aforementioned head-to-head studies [16-18].

\section{Methods}

\section{Setting and study population}

Details on study design have been published elsewhere [16-18]. Briefly, the studies included male or female subjects, aged 18-to-65 years, with a current history of migraine with or without aura, according to International Headache Society (IHS) criteria [24], and with at least one, but no more than 6 migraine attacks per month for the 6 months prior to entering the study. Subjects were recruited in 43 different headache centers in Italy.

Patients could not be enrolled in the study in case of: a) uncontrolled hypertension; b) ischemic heart disease; c) cardiac arrhythmias or symptomatic Wolff-Parkinson-White syndrome; d) previous stroke or cerebral transient ischemic attack; e) severe liver or renal impairment; f) any other severe or disabling medical condition; g) history of alcohol or analgesic or psychotropic drug abuse; h) known hypersensitivity to study drugs; i) previously demonstrated inadequate response to at least two triptans; $j$ ) current use of propranolol or ergot derivatives as prophylactic agents; $k$ ) current use or use in the previous 2 weeks of MAO-inhibitors; l) use of either study medication to treat any one of the last three episodes of migraine; $\mathrm{m}$ ) other headaches lasting for more than 6 days. Pregnant and breast-feeding women were excluded as well, while women with childbearing potential but not using an effective contraceptive method were submitted to a pregnancy test, if clinically indicated.

Written informed consent was obtained from all patients prior to their inclusion into the study. The study was approved by the Independent Institutional Review Boards of the study centers.

\section{Study design}

All studies had a randomized, double blind, crossover design. Each patient received frovatriptan $2.5 \mathrm{mg}$ or rizatriptan $10 \mathrm{mg}$ [16], frovatriptan $2.5 \mathrm{mg}$ or zolmitriptan $2.5 \mathrm{mg}$ [17], and frovatriptan 2.5 mg or almotriptan $12.5 \mathrm{mg}$ [18]. Treatment assignment was made possible through a balanced computer-generated randomized sequence (1:1), where frovatriptan had to be followed by the comparator or vice-versa. Randomization was performed by blocks of 4. Blindness was ensured by the overencapsulation technique, i.e. by inserting study drug tablets in capsules. After treating up to 3 episodes of migraine in no more than 3 months with the first treatment, the patients had to switch to the other treatment and were asked to treat a maximum of three episodes of migraine in no more than 3 months with the second treatment.

The study included a total of three visits and each patient's participation time in the study had not to exceed 6 months from randomization. Subjects having no migraine episodes during either first or second observation period were excluded from the study.

During the randomization visit, after signing written informed consent, subjects provided a detailed general and headache history, and their medication use was evaluated. A physical and neurological examination and pregnancy test (if appropriate) were performed. Blood pressure and heart rate were measured for all subjects. The degree of migraine-associated disability (MIDAS questionnaire) was also measured. At the end of the first visit a headache diary showing characteristics of headache pain and associated symptoms was delivered together with study medication. Subjects were instructed to treat up to 3 migraine episodes occurring in no more than 3 months and to return for the second visit. On this occasion, use of concomitant medications and possible occurrence of adverse events (as gathered from the diary) were evaluated, blood pressure and heart rate were recorded, and a pregnancy test performed, if deemed necessary. The same procedures were carried out at the end of the second study treatment period or at the early withdrawal visit, together with the administration of the patient's preference questionnaire.

Patients were instructed to take one dose of study medication as early as possible after the onset of migraine attack. If insufficient relief had been obtained at 2 hours, patients were allowed to take a second dose of study medication, with a maximum daily intake of two doses. In case of insufficient benefit one hour after the intake of the second dose of the study medication, patients were allowed to take a rescue medication. Alternate rescue medications could not include triptans or ergot alkaloids.

\section{Data analysis}

In the original studies, analysis was performed on the intention-totreat population, including all patients who treated at least one attack in each treatment period and completed the preference questionnaire.

The study endpoints were established following the IHS Guidelines [24]. The objectives were: a) the number of pain-free episodes at 2 hours, i.e. absence of pain within 2 hours of the intake of one dose of study drug and without any rescue medication; b) the number of pain relief episodes at 2 hours, defined as a decrease in migraine intensity from severe or moderate to mild or none, after the intake of one study drug dose; and c) relapse within 48 hours, defined according to the IHS Guidelines, namely when a patient is pain-free within 2 hours after treatment and headache of any severity returns within 48 hours, or requires the use of rescue medication or a second dose of study drug.

Each outcome measure was compared between workday and weekend attacks and within each type of triptan therapy. Continuous variables were summarized by computing average values and standard deviation (SD), while categorical variables by computing the absolute value and the frequency (as percentage). Study endpoints were 
Citation: Lisotto C, Savi L, Pinessi L, Guidotti M, Omboni S, et al. (2014) Efficacy of Frovatriptan vs. Other Triptans in Weekend Migraine: Pooled Analysis of Three Double-Blind, Randomized, Crossover, Multicenter Studies. Brain Disord Ther 3: 128. doi:10.4172/2168-975X. 1000128

Page 3 of 6

compared between weekend and workday attacks by analysis of variance (ANOVA), in case of continuous variables, and by Chisquare test, in case of discrete variables. P-value refers to the statistical significance analyzing between-treatment difference. The level of statistical significance was kept at $<0.05$ throughout the whole study.

\section{Results}

\section{Baseline demographic and clinical data.}

Figure 1 illustrates the patients flow during the study. Out of the total 346 patients, 188 (54\%) of the pooled intention-to-treat population, reported attacks of migraine without aura occurring also on weekends: these 188 patients were treated with frovatriptan (100\%), $62(33 \%)$ with rizatriptan, $60(32 \%)$ with zolmitriptan and $66(35 \%)$ with almotriptan, respectively. Table 1 shows main demographic and clinical characteristics of these patients. Most of the enrolled subjects were females. No statistically significant differences were observed between the main study group and the weekend migraine subgroup as for the clinical features taken into consideration.

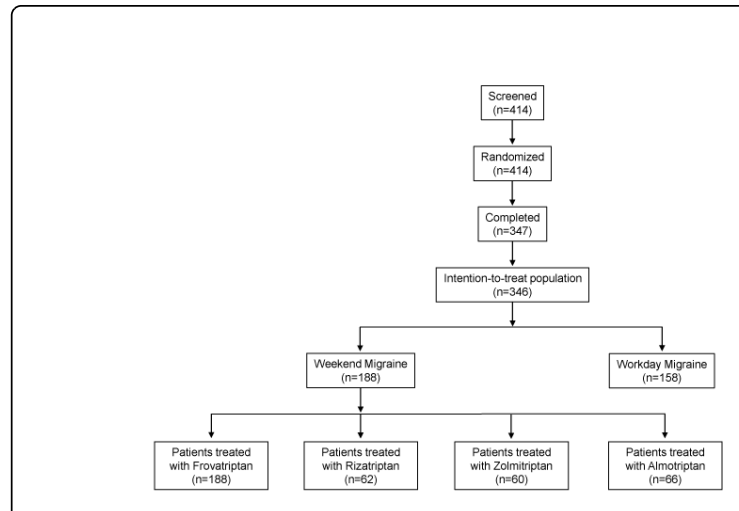

Figure 1: Flow diagram of the patients throughout the study

\begin{tabular}{|c|c|c|c|}
\hline & $\begin{array}{l}\text { Subjects with } \\
\text { weekend migraine } \\
(n=188)\end{array}$ & $\begin{array}{l}\text { Whole study } \\
\text { population } \\
(n=346)\end{array}$ & p-value \\
\hline Age (years, means $\pm S D$ ) & $38 \pm 9$ & $38 \pm 10$ & NS \\
\hline Females (n, \%) & $151(80)$ & $280(81)$ & NS \\
\hline Height $(\mathrm{cm}$, means $\pm \mathrm{SD})$ & $166 \pm 8$ & $166 \pm 8$ & NS \\
\hline Weight $(\mathrm{kg}$, means $\pm \mathrm{SD})$ & $65 \pm 11$ & $64 \pm 12$ & NS \\
\hline Age at onset of migraine (years, means $\pm S D$ ) & $17 \pm 7$ & $17 \pm 7$ & NS \\
\hline Migraine attack duration $>2$ days $(\mathrm{n}, \%)$ & $38(20)$ & $75(22)$ & NS \\
\hline MIDAS score (means $\pm S D$ ) & $23 \pm 15$ & $22 \pm 15$ & NS \\
\hline No use of triptans in the previous 3 months (n, \%) & $51(27)$ & $95(28)$ & NS \\
\hline
\end{tabular}

Table 1: Baseline demographic and clinical data of the 188 patients included in the analysis of weekend migraine and of the 346 subjects of the whole study population. Data are shown as mean $( \pm \mathrm{SD})$, or absolute $(\mathrm{n})$ and relative frequency $(\%)$.

\section{Characteristics of migraine attacks}

A total of 1,850 migraine attacks without aura were recorded during the study periods. Of these attacks, 1,281 (69\%) occurred during workdays and $569(31 \%)$ during weekends. The proportion of weekend attacks was similar for the four treatment periods: $31 \%$ under frovatriptan, $28 \%$ rizatriptan, $31 \%$ zolmitriptan and 33\% almotriptan, respectively. As shown in Figure 2 (top panel), when single days were taken into account, migraine attacks resulted unevenly distributed throughout the week. In regard to the specific day of the week, the average number of attacks was slightly, but significantly $(\mathrm{p}<0.0001)$, greater during the weekends ( $285 \pm 45$ attacks) than during the workdays (256 \pm 37 attacks). The intensity of attacks, recorded immediately before drug intake, was independent of the day of occurrence throughout the week (Figure 2, bottom panel), in particular on weekends compared to workdays $(2.0 \pm 0.7$ vs. $2.0 \pm 0.6$, $\mathrm{p}=\mathrm{NS}$ ).

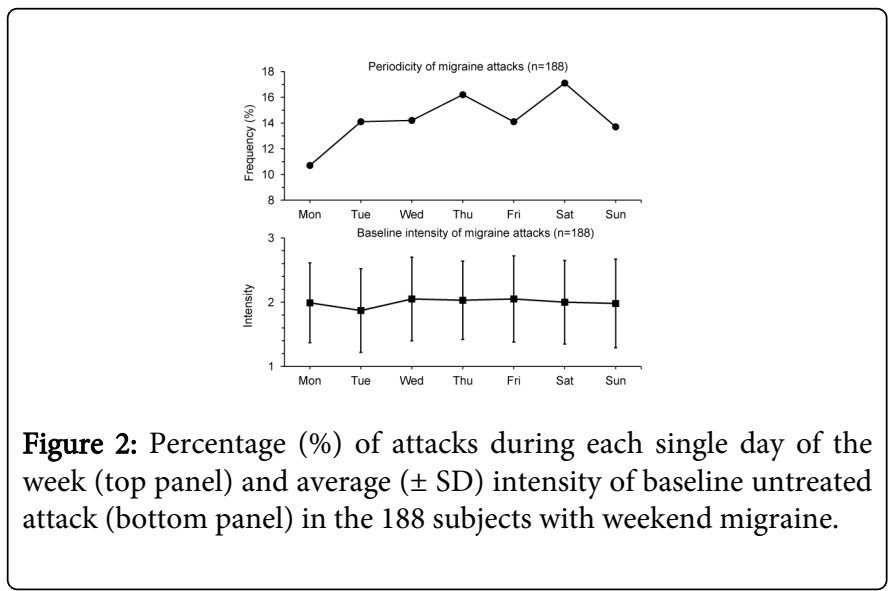




\section{Treatment efficacy of migraine attacks}

In the intention-to-treat population, proportions of pain-free and pain relief episodes at 2 hours were not significantly different between weekend and workday migraine attacks, for any of the study drugs (Figure 3). The proportion of pain-free at 2 hours was $26 \%$ during weekends and $27 \%$ during workdays under frovatriptan, and $34 \%$ and $32 \%$ under the pooled together comparators, respectively. Pain relief episodes were also similarly distributed between weekend and nonweekend attacks for both frovatriptan ( $40 \%$ vs. $42 \%)$ and comparators ( $49 \%$ vs. $43 \%)$.

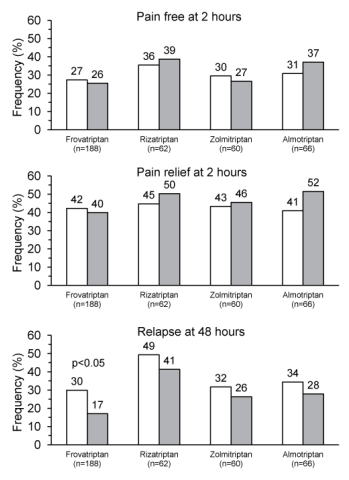

Figure 3: Proportion (\%) of pain free at 2 hours, pain relief at 2 hours and relapse at 48 hours during workday (open bar) and weekend attacks (grey bar) in the 188 subjects with weekend migraine. Data are separately shown for each of the four triptans tested in the study. The p-value refers to statistical significance of the between-period difference.

Conversely, proportion of recurring episodes (relapses) was significantly lower $(\mathrm{p}<0.05)$ under frovatriptan during weekend rather than during workday attacks ( $17 \%$ vs. $30 \%)$, whereas no difference between the two periods was observed for the attacks treated with the other pooled together triptans (weekends $34 \%$ vs. workdays $40 \%$ ), as shown in Figure 3.

\section{Discussion}

In our pooled analysis of three double-blind, randomized, crossover studies, no significant difference was found between weekend and workday attacks treated with frovatriptan and comparators (rizatriptan, zolmitriptan and almotriptan) in terms of pain-free and pain relief rates at 2 hours. When headache relapse within 48 hours was considered, frovatriptan showed a more sustained relieving action compared to the other triptans. As a matter of fact, the relapse rate at 48 hours for attacks occurring on weekends was significantly lower with frovatriptan. This finding is not surprising, since frovatriptan is known to have a long half-life, thus possibly determining a lower proportion of headache recurrence [25-27]. Moreover, this result could be explained by the very early intake of medication, during migraine premonitory phase, due to the consistent predictability of attacks [13]. Interestingly, these data found in the subgroup of subjects treating migraine attacks occurring on weekends, are in line with those observed in the main study population [28] and in the subgroups of women with menstrual migraine [22] and with oral contraceptiveinduced menstrual migraine [29].

Our retrospective analysis of the three double-blind, randomized studies represents the first study assessing the efficacy of different triptans in the acute treatment of weekend migraine. Actually there are other studies which specifically evaluated the effectiveness of the treatment of weekend migraine with triptans. In an open-label, nonrandomized study 28 subjects suffering from weekend migraine were prophylactically treated for two consecutive weekends with frovatriptan $2.5 \mathrm{mg}$, followed by two other consecutive weekends with naproxen $500 \mathrm{mg}$ [30]. Both medications were taken in a single dose on Saturday and Sunday morning. Active therapy was preceded by two weekends during which no treatment was administered. The treatment with frovatriptan was associated with a lower migraine intensity, particularly in the subgroup of patients not taking any rescue medication. In addition, the use of rescue medication was lower under frovatriptan than naproxen. The second study assessed the short-term efficacy of rizatriptan $5 \mathrm{mg}$ vs. placebo in 476 adolescents with migraine [31]. In this single-attack study, patients were instructed to use the study medication only on non-school days. Even if fewer patients than expected (30\%) treated their migraine attacks on weekends, the proportion of pain relief at 2 hours was significantly higher in the rizatriptan group than in the placebo group ( $74 \%$ vs. $58 \%$ ). In an open-label, prospective, observational study which included 200 patients with weekend migraine, 99 subjects were treated with a short-term prophylaxis either with magnesium (63 cases) or with frovatriptan $2.5 \mathrm{mg}$ (36 cases). Patients with weekend migraine were arbitrarily defined as subjects with at least $75 \%$ of all their migraine attacks occurring on weekends, with a frequency ranging from 1 attack every 2 months to 4 attacks per month. Five doses were administered, one on Friday evening and on Sunday morning, and two (one in the morning and one in the evening) on Saturday. The endpoint was the reduction by at least $50 \%$ in headache hours. This decrease was noted in $9.5 \%$ of patients taking magnesium and in $63.9 \%$ of subjects treated with frovatriptan $2.5 \mathrm{mg}$ [13].

Our study provides some additional matter of discussion. First, we did not find a particular difference in migraine intensity between weekend and workday attacks, which confirms previous evidence [8]. Second, although the study did not specifically enroll weekend migraineurs, the average number of migraine attacks was significantly higher during days off rather than during workdays, confirming the possible triggering role of relaxation after stress to provoke migraine attacks $[2,8]$. Third, we assert that weekend migraine cannot be considered as a distinct subtype of migraine. Weekends, as well as stress, some foods and beverages, sleep (too much or too little), weather changes and several other factors just act as triggers. Interestingly, a significant number of migraine patients report their attacks to occur mostly on weekends, as if they were susceptible almost only to this factor. Nonetheless, weekend migraine does not seem to occur so consistently as menstrually related migraine, the only subtype of migraine included in the International Classification of Headache Disorders-3 beta Appendix. Therefore, we did not propose any diagnostic criteria for weekend migraine, since we believe that it cannot be considered as a possible distinct entity, as likely menstrually related migraine. We deem that more attention should be given to weekend migraine in future studies, in order to establish a strict and clinically acceptable definition, with clear diagnostic elements, suitable for clinical practice e for possible future investigations on this type of migraine. Fourth, previous studies have shown that, due to its pharmacological properties, frovatriptan is particularly effective in the 
short-term prophylactic therapy of some types of migraine, like menstrually related migraine [32-34] and long-lasting migraine attacks, usually with a high relapse rate [35]. In 2012 a Task Force of the American Academy of Neurology and the American Headache Society published the revised clinical practice guidelines for episodic migraine prevention in adults. Frovatriptan was the only triptan included in these guidelines, notably in the level A medications group, labelled with the specific indication for short-term prophylaxis of menstrually related migraine, based on two class I studies [36].

Given that weekend migraine is also characterized by consistent triggering factors and a predictable occurrence, a possible benefit of frovatriptan as prophylactic agent as well as symptomatic medication of this subtype of migraine should be explored in the future.

Some weaknesses of our study deserve to be discussed. First, we performed a retrospective analysis on a subgroup of patients, who were not originally selected as specifically weekend migraine sufferers. Although we included a large number of patients $(n=188)$ and attacks $(n=569)$, a post-hoc power analysis, run in this subgroup of subjects, revealed differences in statistical power between frovatriptan (98\%) and the comparators pooled together (70\%). Albeit the sample size might have been inadequate, our results appear to be clinically relevant, because no such prospective studies comparing the efficacy of different triptans in weekend migraineurs have yet been carried out. In addition, baseline characteristics of our subgroup of migraineurs were similar to those of the main study population. We also limited as much as possible the number of endpoints and outcome measures, and the study assumptions, since the evaluation of this subgroup of migraine patients can be considered as a benchmark for subsequent future clinical trials to confirm or refute our findings. Finally, the proportion of subjects having migraine attacks on weekends (54\%) was slightly higher than that reported in the literature $(20-30 \%)(1,4)$. Thus far, this might be probably related to the bias in the selection of patients and to the retrospective nature of our analysis.

In conclusion, the results of our systematic analysis of individual data of three double-blind, randomized, direct, comparative studies provide strong evidence that triptans are effective also in treating weekend migraine attacks. Among the different triptans, frovatriptan seems to offer the advantage of a lower risk of relapse and therefore a more sustained effect during attacks occurring on days off from work. Moreover, frovatriptan can be used as a symptomatic medication, but also prophylactically for a short-term preventive treatment. We acknowledge that further large double-blind, randomized, studies are needed and warranted to explore the possible acute and prophylactic efficacy of frovatriptan in patients specifically suffering from weekend migraine.

\section{Acknowledgements}

The present study was supported by Istituto Lusofarmaco d'Italia.

\section{Conflict of Interest}

All authors have occasionally served as scientific consultants for manufacturers of frovatriptan, rizatriptan, zolmitriptan or almotriptan. Deborha Pezzola and Dario Zava are employees of Istituto Lusofarmaco d'Italia S.p.A.

\section{References}

1. Couturier EG, Hering R, Steiner TJ (1992) Weekend attacks in migraine patients: caused by caffeine withdrawal? Cephalalgia 12: 99-100.

2. Torelli P, Cologno D, Manzoni GC (1999) Weekend headache: a retrospective study in migraine without aura and episodic tension-type headache. Headache 39: 11-20.

3. Cugini P, Romit A, Di Palma L, Giacovazzo M (1990) Common migraine as a weekly and seasonal headache. Chronobiol Int 7: 467-469.

4. Torelli P, Cologno D, Manzoni GC (1999) Weekend headache: a possible role of work and life-style. Headache 39: 398-408.

5. Wöber C, Brannath W, Schmidt K, Kapitan M, Rudel E, et al. (2007) Prospective analysis of factors related to migraine attacks: the PAMINA study. Cephalalgia 27: 304-314.

6. Morrison DP (1990) Occupational stress in migraine--is weekend headache a myth or reality? Cephalalgia 10: 189-193.

7. Alstadhaug KB, Salvesen R, Bekkelund S (2007) Weekend migraine. Cephalalgia 27: 343-346.

8. Nattero G, De Lorenzo C, Biale L, Allais G, Torre E, et al. (1989) Psychological aspects of weekend headache sufferers in comparison with migraine patients. Headache 29: 93-99.

9. Evers S, Afra J, Frese A, Goadsby PJ, Linde M, et al. (2009) EFNS guideline on the drug treatment of migraine--revised report of an EFNS task force. Eur J Neurol 16: 968-981.

10. Loder E (2010) Triptan therapy in migraine. N Engl J Med 363: 63-70.

11. Johnston MM, Rapoport AM (2010) Triptans for the management of migraine. Drugs 70: 1505-1518.

12. Belvís R, Pagonabarraga J, Kulisevsky J (2009) Individual triptan selection in migraine attack therapy. Recent Pat CNS Drug Discov 4: 70-81.

13. Evers S, Lisotto C (2013) An algorithm of migraine treatment. European Neurological Review 8: 149-152.

14. Balbisi EA (2004) Frovatriptan succinate, a 5-HT1B/1D receptor agonist for migraine. Int J Clin Pract 58: 695-705.

15. Sanford M (2012) Frovatriptan: a review of its use in the acute treatment of migraine. CNS Drugs 26: 791-811.

16. Savi L, Omboni S, Lisotto C, Zanchin G, Ferrari MD et al. (2011). A double-blind, randomized, multicenter, Italian study of frovatriptan versus rizatriptan for the acute treatment of migraine. J Headache Pain 12: 219-226.

17. Tullo V, Allais G, Ferrari MD, Curone M, Mea E, et al. (2010) Frovatriptan versus zolmitriptan for the acute treatment of migraine: a double-blind, randomized, multicenter, Italian study. Neurol Sci 31 Suppl 1: S51-54.

18. Bartolini M, Giamberardino MA, Lisotto C, Martelletti P, Moscato D et al. (2011). A double-blind, randomized, multicenter, Italian study of frovatriptan versus almotriptan for the acute treatment of migraine. J Headache Pain 12: 361-368.

19. Savi L, Omboni S, Lisotto C, Zanchin G, Ferrari MD et al. (2011). Efficacy of frovatriptan in the acute treatment of menstrually related migraine: analysis of a double-blind, randomized, cross-over, multicenter, Italian, comparative study versus rizatriptan. J Headache Pain 12: 609-615.

20. Allais G, Tullo V, Benedetto C, Zava D, Omboni S et al. (2011) Efficacy of frovatriptan in the acute treatment of menstrually related migraine: analysis of a double-blind, randomized, multicenter, Italian, comparative study versus zolmitriptan. Neurol Sci 32: S99-S104.

21. Bartolini M, Giamberardino MA, Lisotto C, Martelletti P, Moscato D et al. (2012) Frovatriptan versus almotriptan for acute treatment of menstrual migraine: analysis of a double-blind, randomized, cross-over, multicenter, Italian, comparative study. J Headache Pain 13: 401-406.

22. Allais G, Tullo V, Omboni S, Benedetto C, Sances G et al. (2012) Efficacy of frovatriptan versus other triptans in the acute treatment of menstrual migraine: pooled analysis of three double-blind, randomized, crossover, multicenter studies. Neurol Sci 33: S65-S69. 
Citation: Lisotto C, Savi L, Pinessi L, Guidotti M, Omboni S, et al. (2014) Efficacy of Frovatriptan vs. Other Triptans in Weekend Migraine: Pooled Analysis of Three Double-Blind, Randomized, Crossover, Multicenter Studies. Brain Disord Ther 3: 128. doi:10.4172/2168-975X. 1000128

Page 6 of 6

23. Tullo V, Allais G, Curone M, Ferrari MD, Omboni S et al. (2012) Frovatriptan versus zolmitriptan for the acute treatment of migraine with aura: a subgroup analysis of a double-blind, randomized, multicenter, Italian study. Neurol Sci 33: S61-S64.

24. Headache Classification Subcommittee of the International Headache Society. The International Classification of Headache Disorders, 2nd ed. Cephalalgia (2004) 24: 9-160.

25. Kelman L (2008) Review of frovatriptan in the treatment of migraine. Neuropsychiatr Dis Treat 4: 49-54.

26. Géraud G, Keywood C, Senard JM (2003) Migraine headache recurrence: relationship to clinical, pharmacological, and pharmacokinetic properties of triptans. Headache 43: 376-388.

27. Negro A, Lionetto L, Casolla B, Lala N, Simmaco M, et al. (2011) Pharmacokinetic evaluation of frovatriptan. Expert Opin Drug Metab Toxicol 7: 1449-1458.

28. Cortelli P, Allais G, Tullo V, Benedetto C, Zava D et al. (2011) Frovatriptan versus other triptans in the acute treatment of migraine: pooled analysis of three double-blind, randomized, cross-over, multicenter, Italian studies. Neurol Sci 32: S95-S98.

29. Allais G, Tullo V, Omboni S, Pezzola D, Zava D et al. (2013) Frovatriptan vs. other triptans for the acute treatment of oral contraceptive-induced menstrual migraine: pooled analysis of three double-blind, randomized, crossover, multicenter studies. Neurol Sci 34: S83-S86.

30. Guidotti M, Barrilà C, Leva S, De Piazza C, Omboni S (2013) Symptomatic or prophylactic treatment of weekend migraine: an open- label, nonrandomized, comparison study of frovatriptan versus naproxen sodium versus no therapy. Neuropsychiatr Dis Treat 9: 81-85.

31. Visser WH, Winner P, Strohmaier K, Klipfel M, Peng Y et al. (2004) Rizatriptan Protocol 059 and 061 Study Groups. Rizatriptan $5 \mathrm{mg}$ for the acute treatment of migraine in adolescents: results from a double-blind, single-attack study and two open-label, multiple-attack studies. Headache 44: 891-899.

32. Balbisi EA (2006) Frovatriptan: a review of pharmacology, pharmacokinetics and clinical potential in the treatment of menstrual migraine. Ther Clin Risk Manag 2: 303-308.

33. Brandes JL, Poole Ac, Kallela M, Schreiber CP, MacGregor EA, et al. (2009) Short-term frovatriptan for the prevention of difficult-to-treat menstrual migraine attacks. Cephalalgia 29: 1133-1148.

34. Silberstein SD, Berner T, Tobin J, Xiang Q, Campbell JC (2009) Scheduled short-term prevention with frovatriptan for migraine occurring exclusively in association with menstruation. Headache 49: 1283-1297.

35. Kelman L, Harper SQ, Hu X, Campbell JC (2010) Treatment response and tolerability of frovatriptan in patients reporting short- or longduration migraines at baseline. Curr Med Res Opin 26: 2097-2104.

36. Silberstein SD, Holland S, Freitag F, Dodick DW, Argoff C et al. (2012) Evidence-based guideline update: Pharmacologic treatment for episodic migraine prevention in adults. Report of the Quality Standards Subcommittee of the American Academy of Neurology and the American Headache Society. Neurology 78: 1337-1345. 